\title{
Two Cobalt(II) 5-Aminoisophthalate Complexes and Their Stable Supramolecular Microporous Frameworks
}

\author{
En Tang ${ }^{a}$, Yu-Mei Dai ${ }^{a, b}$, Jian Zhang $^{a}$, Zhao-Ji Li $^{a}$, Yuan-Gen Yao ${ }^{a}{ }^{*}$, Jie Zhang $^{a}{ }^{*}$ \\ and Xu-Dong Huang ${ }^{c}$
}

The magnetic behavior of the dehydrated product of 2: figure s1 and figure s2.

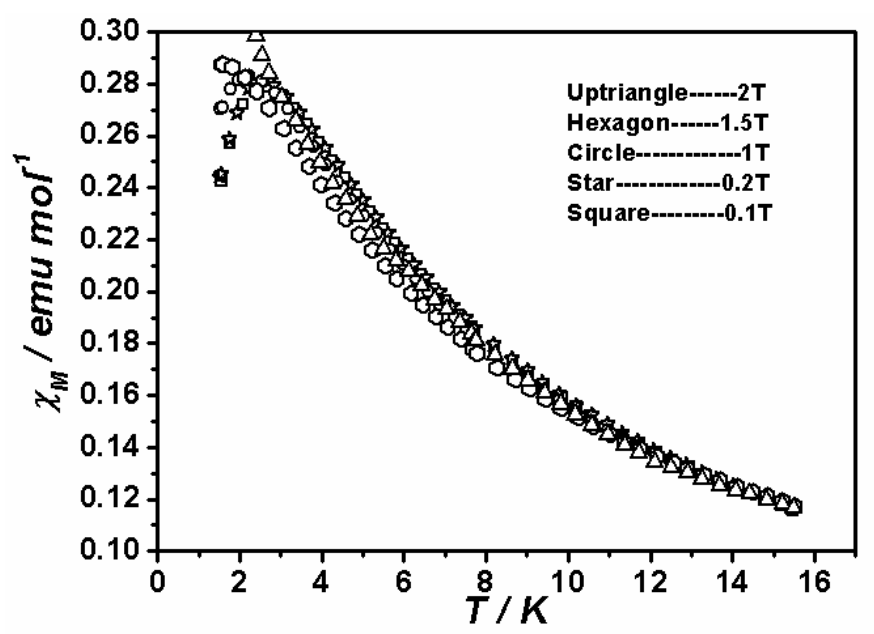

Fig. S1 Evolution of the magnetic susceptibility of dehydrated $\mathbf{2}$ as a function of the temperature, measured on cooling under external magnetic field (2T, $1.5 \mathrm{~T}, 1 \mathrm{~T}, 0.2 \mathrm{~T}, 0.1 \mathrm{~T}$, from top to bottom in the plot) which are noted on the graph. 


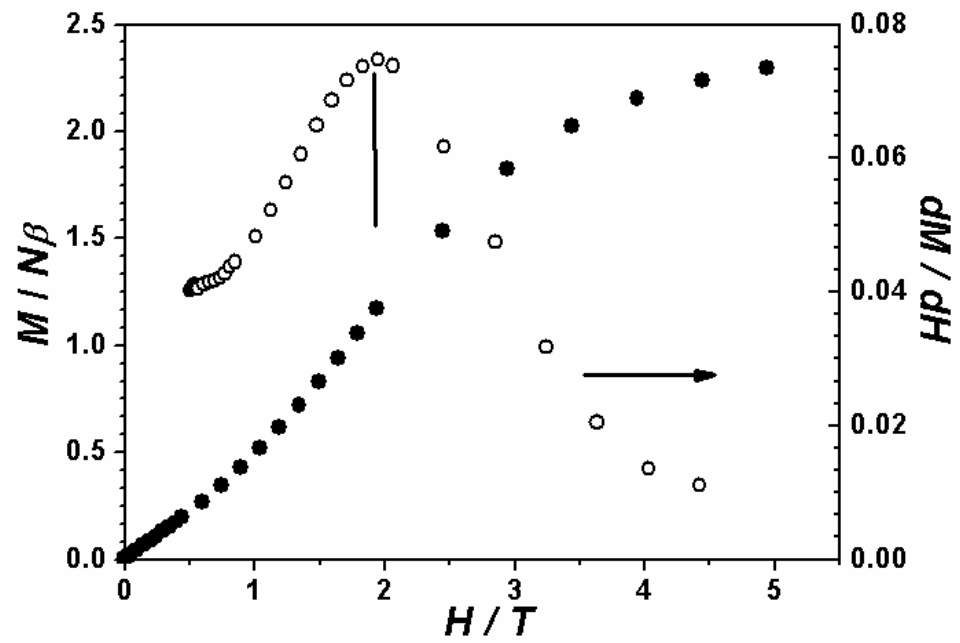

Fig. S2 Plots of the field-dependent magnetization of dehydrated 2 at $2 \mathrm{~K}$ with the critical field being of ca. $2 \mathrm{~T}$. 